\title{
From Tactile to NavTile: Opportunities and Challenges with Multi-Modal Feedback for Guiding Surfaces during Non-Visual Navigation
}

\author{
Saiganesh Swaminathan* \\ Human-Computer Interaction \\ Institute, Carnegie Mellon University \\ Pittsburgh, PA, USA
}

\author{
Yellina Yim \\ Human-Computer Interaction \\ Institute, Carnegie Mellon University \\ Pittsburgh, PA, USA
}

\author{
Scott E Hudson \\ Human-Computer Interaction \\ Institute, Carnegie Mellon University \\ Pittsburgh, PA, USA
}

\author{
Cynthia L Bennett \\ Human-Computer Interaction \\ Institute, Carnegie Mellon University \\ Pittsburgh, PA, USA
}

\author{
Patrick Carrington \\ Human-Computer Interaction \\ Institute, Carnegie Mellon University \\ Pittsburgh, PA, USA
}

\begin{abstract}
Tactile guiding surfaces in the built environment have held a contentious place in the process of navigation by people who are blind or visually impaired. Despite standards for tactile guiding surfaces, problems persist with inconsistent implementation, perception, and geographic orientation. We investigate the role of tactile cues in non-visual navigation and attitudes surrounding guiding surfaces through a survey of 67 people with vision impairments and ten interviews with navigation and public accessibility experts. Our participants revealed several opportunities to augment existing tactile surfaces while envisioning novel multimodal feedback solutions in immediately relevant contexts. We also propose an approach for designing and exploring low cost, multimodal tactile surfaces, which we call navtiles. Finally, we discuss practical aspects of implementation for new design alternatives such as standardization, installation, movability, discoverability, and a need for transparency. Collectively, these insights contribute to the production and implementation of novel multimodal navigation aids.
\end{abstract}

\section{CCS CONCEPTS}

- Human-centered computing $\rightarrow$ Accessibility; • Social and professional topics $\rightarrow$ People with disabilities.

\section{KEYWORDS}

Navigation, Tactile, Blind, Low-Vision, Textured Surface, Mobility

ACM Reference Format:

Saiganesh Swaminathan, Yellina Yim, Scott E Hudson, Cynthia L Bennett, and Patrick Carrington. 2021. From Tactile to NavTile: Opportunities and

${ }^{*}$ Contact Author Email: saiganes@cs.cmu.edu

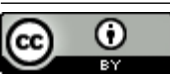

This work is licensed under a Creative Commons Attribution International 4.0 License.

CHI '21, May 8-13, 2021, Yokohama, Japan

(c) 2021 Copyright held by the owner/author(s).

ACM ISBN 978-1-4503-8096-6/21/05.

https://doi.org/10.1145/3411764.3445716
Challenges with Multi-Modal Feedback for Guiding Surfaces during NonVisual Navigation. In CHI Conference on Human Factors in Computing Systems (CHI '21), May 8-13, 2021, Yokohama, Japan. ACM, New York, NY, USA, 13 pages. https://doi.org/10.1145/3411764.3445716

\section{INTRODUCTION}

Mobility has transformed over the years with the availability of navigational technologies. Many of these innovations help with non-visual navigation assistance. These tools collect information through computer vision, GPS, robotics, crowd work, and more to provide cues in a variety of mediums from audio to haptics. However, studies [45] have shown tactile features from the built environment remains an important, versatile, and underutilized perceptual cue. Orientation and Mobility instructors train non-visual navigators to utilize many tactile cues from indoor and outdoor environments [22]. Well established techniques such as shorelining, trailing the wall, and more enable students to recognize cues from walls, the grassline on the pavement, and railings with great success. Furthermore, navigators can leverage cues from objects such as fences, mailboxes, traffic signs, fire hydrants, or benches while also detecting changes in terrain.

While many such natural tactile cues exist in the built environment and are used by non-visual navigators, these cues are often not reliable as they can be discrete (not continuous) and available only for short durations while navigating. As a result, the built environment is outfitted with purposefully installed tactile guiding surfaces that exist in various tactile patterns to support non-visual navigators with warnings, alerts, and guidance. Tactile guiding surfaces in the built environment, however, have held a contentious place in the process of supporting navigation by people who are blind or visually impaired. Despite the relatively small set of individual textures, the standards for tactile guiding surfaces are vague and the implementation of pavement is not consistent [37]. This often leads to confusing situations for non-visual navigators, and in extreme cases, even injuries. Also, the information conveyed through tactile guidance surfaces is prone to cross-talk with other environmental noise such as foot traffic and has detectability issues due to weather conditions like snow or mud [23,30]. Finally, most prominently, they do not help with geographic orientation during wayfinding, i.e, the tactile guidance surfaces inherently lack the 
ability to determine position relative to the final destination, topography, or distance in an unfamiliar area [9]. While such gaps exist when using tactile guidance surfaces, these gaps could be potentially alleviated by further exploration in design and augmenting the existing tactile guidance surfaces with additional modalities such as audio or vibrations [32].

In this paper, we begin by reviewing key literature on nonvisual navigation, multi-modal feedback for mobility, and challenges with tactile guiding surfaces. We then describe our mixed-methods investigation with a survey of 67 blind or visually impaired travelers as well as interviews with 10 orientation \& mobility (O\&M) instructors and public accessibility experts. We share results about the role of tactile cues in everyday non-visual navigation and attitudes surrounding the use of guiding surfaces. Our participants revealed several opportunities for augmenting existing tactile surfaces with novel multimodal feedback solutions in immediately relevant contexts. We discuss insights from experts on practical aspects of implementation and critical issues extending beyond new design alternatives such as standardization, installation, movability, discoverability, and a need for transparency.

Finally, we offer a potential approach for rapidly creating lowcost multi-modal tactile surfaces with easily available materials and tools \& techniques to widen the process of production. We highlight opportunities for augmenting tactile surfaces using available low-cost sensors and to amplify tactile feedback with audio. Taken together, our insights from key stakeholders - blind and visually impaired users, O\&M instructors, and public accessibility experts - contribute to an understanding of how novel multi-modal navigational aids may be better designed and integrated into the built environment.

\section{RELATED WORK}

In this section, we overview the literature on nonvisual navigation, the use of multi-modal messaging to support nonvisual sensemaking, and tactile surface production method. Together, these topics frame the foundation of the research we build upon--fabrication to augment tactile cues.

\subsection{Non-Visual Navigation using social cues and built environment}

Researchers have observed blind people, and sometimes their travel companions while navigating public places. They found that blind people triangulate several cues into their navigation and exploration practices. For example, blind people combine information gathered from a multitude of sources to decide the next steps, confirm they are on the right path or reorient their route. These cues are often appropriate features of the built environment not intended to be useful for nonvisual navigation. Williams et al. [44] [46], and Thieme et al. [41], detailed that nonvisual navigators may listen, find objects with their canes, and tap into their memories and collective intelligence when traveling in groups to build a mental map of their surroundings. Crucially, researchers Szpiro et al [36] and Zhao et al. [48] have pointed out that nonvisual navigation occurs on a spectrum with many people who have low vision incorporating their remaining vision into wayfinding. Williams et al. [45] uncovered that many of the techniques used by blind navigators are misunderstood by sighted passersby or companions to be missteps. For instance, tapping an object with a cane was perceived as a mistake by sighted onlookers, but blind navigators often intentionally sought contact with certain objects they had landmarked to confirm they were heading in the intended direction. Mismatched expectations about how blind people should navigate contributed to miscommunications and frustration among travel companions with mixed visual abilities.

Researchers have taken these insights to develop new technologies [18] [4] and information sources to better support nonvisual navigation. A key challenge of making public transportation more nonvisually accessible is locating the desired transit stop and waiting to be noticed by transit drivers. Researchers in this area have recognized the role of built environments in providing important cues. However, blind people traveling in unfamiliar environments may not know what cues to even look for. As such, Hara et al. [20] and Campbell et al. [12] have leveraged crowdsourcing from the public and information sourcing from public transit agency databases to clue riders ahead of time of the types of landmarks they can expect near their transit stop. These studies revealed the importance of accuracy, independence, and safety in context. Having access to information about landmarks increased participants' sense of independence, as it leveraged existing features of the built environment and the common practice of nonvisual landmarking to ease bus stop search. Information sources necessary for crowdsourcing however can be inaccurate, and maintaining requires ongoing support. Our present study instead explores possibilities for outfitting the built environment.

\subsection{Multi-Modal Feedback for Navigation and Mobility}

Previous research has examined how multimodal feedback has helped with navigation and mobility. In general, there are two approaches to offering multi-modal feedback. The first is to provide training with audio-tactile solutions before actually engaging in the navigation. For instance, Homere [26] is a cane controller connected to a robot arm system that provides auditory, tactile, and thermal feedback for blind and visually impaired users to get a simulation of texture and collision in the virtual world. Similarly, Blind Aid [33] simulates a virtual world by using a phantom to simulate force feedback generated by a white cane during navigation and provides auditory feedback. While some researchers designed haptic systems to experience real-world forces in the built environment, Thevin et al. [38] and Kunz et al. [24] designed virtual reality environments for blind and low vision people to train indoors.

The more recent canetroller project [47] provides auditory and haptics through cane simulations for acquainting blind users with real-world objects and textures. Talking tactile tablet [25] is another project which enables spatial understanding of content by providing auditory feedback as users explore tactile content such as maps. While the use of audio-tactile content is useful, researchers have further explored how to enable sighted users to create tactile content with augmented reality [39] [40].

Additionally, Another body of work has also examined how to provide multi-modal feedback during navigation. The virtual 
leading blocks [5] project examined a floor instrumented with RFids and provided tactile 'feedback on the go' to guide users. Similarly, CyberGrasp [43] is a haptic glove that generates force feedback to blind user's fingers for navigating the virtual space with a cane while providing audio feedback.

\subsection{Tactile Surface Production and Application}

Tactile surface production is most often studied to make nonvisual instruction in STEM more accessible. For example, braille embossers are commonly used to create tactile graphics; however, they are expensive, costing upwards of $\$ 5000$, [3], and generating such highquality graphics requires expertise. Other methods widely used include swell form graphics paper [2]; marked surfaces of the paper raise when heated. Vacuum/thermoforming [14], which has been widely used in the community for producing copies of relief picture books that are static, is a method more closely related to our work This method, however, still requires the positives to be produced by another process and the machine itself remains expensive due to limited adoption. More recently, due to the widespread availability of low-cost 3D printers (<\$99), DIY technologies have been used for the design and development of assistive technology for people with visual impairments $[11,13,15,21]$. However, to the best of our knowledge, the use of DIY open-source designs of desktop vacuum formers [1] their utility for blind people has not been explored much outside educational contexts, and there remains a high barrier of entry to use 3D modeling software. We leverage both these technologies for prototyping as a complementary method in our work.

\subsection{Standards and Implementation Challenges with Tactile surfaces}

2.4.1 Standards and implementation challenges. Research has shown that properly maintained, designed, produced, and installed tactile surface indicators have proved their potential to provide safety information reliably. ([6]; Barker et al. [7]; DETR [42]; Bentzen et al. [8]; Ståhl et al. [35], Scott et al. [34]). The maintenance and proper implementation of tactile paving surfaces, however, is difficult and practitioners have faced numerous challenges. For instance, standards are often not very detailed, and specific recommendations on how pavements should be designed to fit the broader environment are often missing [37]. Furthermore, the excessive desire of designers to increase the grammar of syntactical use of the tactile pavings has led to the proliferation of a wide variety of patterns, usage and often in situations that are not consistent within the same city [29]. The issue is further exacerbated when implemented across different countries. Several examples of such problems can be seen [29] in figure 1, where tactile pavings located in Paris, Beijing, or Taipei are all incorrectly implemented. Some tactile surfaces are installed with more emphasis on aesthetics than safety leading to sub-optimal use. Other examples of improper use include: When the height of the stairs is less than $2100 \mathrm{~mm}$, it's safer to warn nonvisual navigators with guardrails or seating rather than use tactile paving. In a similar vein, the curb is a better indicator of the edge of sidewalks than tactile surfaces [30].

2.4.2 Survey Procedure. The survey was administered through Qualtrics and was available for three weeks. The survey link was
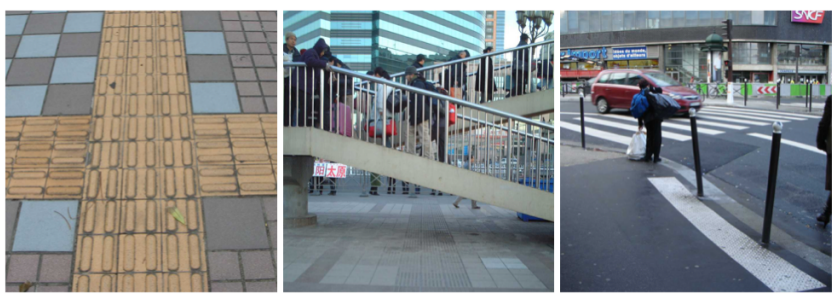

Figure 1: (A) Shows two guidance surfaces intersecting without warning in (Taipei, Taiwan) (B) shows a guidance surface leading to stairs without warning in (Beijing, China) (C) shows Incorrect positioning at the entrance to a crosswalk (Paris, France), all example images referenced from [29]

shared through Twitter and in two closed Facebook Groups for people with disabilities. We collected over 100 responses. We removed responses with erroneous data (e.g. copy-paste answers, incomprehensible text) and excessively incomplete responses (blank sections, multiple skipped questions, etc.). This resulted in 67 completed responses.

The survey consisted of five sections and took 30 minutes to complete. The five sections are listed below:

- Demographics

- Navigation Background -inquiring Tools and Cues used for navigation

- Knowledge of Common textured Surfaces installed to assist nonvisual Navigation.

- Descriptive and qualitative experiences encountering Surfaces in different Contexts.

- Brainstorming new types of textured surfaces and accompanying multimodal feedback.

2.4.3 Survey Participants. We summarize responses from the first two sections here to introduce the background of survey respondents. We describe the remaining survey findings in Section 4 . We received 67 responses to the survey. Of the respondents, 41 identified as female, 24 as male, and 1 person as transgender/agender. The majority of participants were blind ( $55 \%$ ) as opposed to visually impaired or low-vision (41\%). The remaining respondents did not specify their vision impairment. The majority of participants had their vision impairment since birth (65\%) while $25 \%$ had their impairment for more than five years. The majority of survey respondents $(43,64 \%)$ reported primarily navigating in urban environments, while $13(19 \%)$ and $6(9 \%)$ indicated primarily navigating in suburban and rural environments, respectively. The majority of survey participants $(52,78 \%)$ engage in unfamiliar navigation either once or a few times per month, while 13 (19\%) participants reported unfamiliar navigation once or a few times per week. Only two participants indicated daily unfamiliar navigation.

We asked participants to indicate their current use of and preferences for common tools and cues supporting navigation from a set of 14 options. The most used navigation tools were a white cane, smartphones, GPS, audio cues, human assistance, and tactile cues. The least used tools, indicated by a majority of responses having never used them, including guide dogs, wearables, and visual cues (asked for people with some useable vision). The frequency of 
use of vibration cues from the environment, like haptic feedback emitted by some accessible pedestrian signals (APS), was more evenly distributed across "never", "monthly", and "weekly" categories. When asked what tools participants would prefer to use if they had unlimited access, participants indicated the most preferred tools were the white cane, smartphone, audio cues, and tactile cues.

\subsection{Interviews with Public Access and Mobility Experts}

To complement our survey findings, we interviewed five O\&M instructors and five public accessibility experts. According to the information service VisionAware ${ }^{1}$, O\&M refers to the instruction of safe, efficient, and effective travel techniques to people with vision impairments. As such, we recruited O\&M instructors for their expertise in teaching nonvisual navigation to help us situate our survey participants' experiences and suggestions in best practices and safety precautions. The public accessibility experts were recruited as we recognize that public implementation would require collaborative efforts of many stakeholder groups along with developing an in-depth knowledge of the ecosystems into which we would implement novel textured surfaces.

2.5.1 Interview Participants. We recruited five O\&M instructors, four of whom were blind themselves. The fifth (O3) was sighted and had 33 years of experience teaching deaf-blind people, a population that may uniquely benefit from tactile feedback. The remaining four instructors all had experience teaching deaf-blind students and students with other disabilities in addition to blindness, though most of their comments considered nonvisual navigation that assumed normed hearing, spatial, proprioceptive (interpreting bodily movements and position in space), and cognitive abilities.

We recruited five public accessibility experts, two of whom were blind (T1, T5), one had a low vision (T3), and the last two were sighted, one of whom (T2) had physical disabilities and chronic illnesses. We note that O3's advocacy experience meant his expertise overlapped. They brought a diversity of experiences exploring, informing, and implementing accessibility features in public places. This expertise included enthusiastic and intentional travel to nonvisually explore new public space (T1, T3, T5), experience working at public transit agencies to evaluate, implement, and train people to use accessibility features of public transit systems (T1, T5), consulting for government and corporate-funded public place and transit hub redesigns (T3, T4, O3), experience advocating governments for more accessible public places (T1, T2, T3, T5, O3), and experience reviewing crosswalk redesign plans for ADA compliance for a municipality (T4).

2.5.2 Interview Procedure and Analysis. Each interview was conducted via phone or video call and took approximately 1 hour Interviews with O\&M instructors comprised an overview of their job responsibilities, what they teach their students about tactile feedback and an ideation session about potential use cases for new or more consistently implemented existing textured surfaces. Interview questions to public accessibility experts inquired a description of their relevant employment and advocacy, experiences with textured surfaces, and an ideation session about new textured surfaces. All interviews were recorded, transcribed, and thematically analyzed according to the interview questions [10].

\section{ENCOUNTERING TEXTURED SURFACES IN THE WILD}

In this section, we discuss findings from both the survey and expert interviews regarding the current role of textures and attitudes toward their use in non-visual navigation.

\subsection{Knowledge of Available Textured Surfaces}

We asked survey participants to indicate how they detected textured surfaces during navigation. Participants perceived textured surfaces through varying combinations of their hands (66\%), feet $(88 \%)$, and white cane $(87 \%)$. Over half $(51 \%)$ of survey respondents indicated using all three.

Through the survey, we also asked participants to indicate whether they had encountered and recognized common, purposefully-installed textured surfaces. The tactile pattern in surfaces applied depends on the context of its use, for instance, there are patterns that are more applicable in underground metros more than in sidewalks.

In our work presented here we asked participants about four different textured surfaces (see Fig 2) used in the US:

- Blister: Rows of round raised bumps, with flat tops and which are arranged in a square.

- Offset Blister: Rows of round raised bumps, arranged where each row is offset from the next.

- Along Stripes/Cycleway: Flat-topped bars run parallel to the direction of pedestrian traffic.

- Hazard: Flat top bars which are perpendicular to the direction of pedestrian traffic.

The variations in these patterns are both geometrical and based on the arrangement of textures in spaces. For instance, a hazard surface is a series of parallel rectangular bars placed perpendicular to the walking direction where the Guidance strips consist of rectangular bars parallel to the walking direction.

A summary of responses $(n=67)$ for the frequency of encounters for each variation of tactile surfaces is given in Table 1 . The table also summarizes the understanding of each surface. We asked participants to describe the type of information texture patterns were meant to convey. We counted responses that match the purpose outlined by the ISO standard for tactile surface indicators as understanding the meaning of the surface.

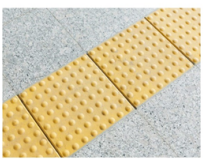

Blister

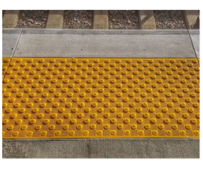

Offset Blister

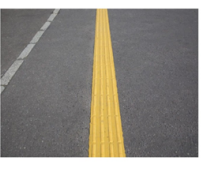

Along Stripes/cycleway

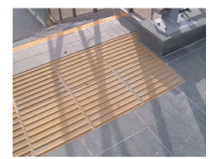

Hazard
Figure 2: Shows most commonly used tactile guidance surfaces for non visual navigation 
Table 1: Frequency of encounters with common surface textures.

\begin{tabular}{|c|c|c|c|c|c|}
\hline Surface Texture Pattern & Never/Less than Monthly & Multiple or once per month & Multiple or once per week & Multiple or once per day & Understood Texture Meaning \\
\hline Blister & 14 & 23 & 13 & 17 & 36 \\
\hline Offset Blister & 45 & 9 & 9 & 3 & 8 \\
\hline Along Stripes/Cycleway & 58 & 3 & 6 & 0 & 8 \\
\hline Hazard & 56 & 5 & 4 & 1 & 2 \\
\hline
\end{tabular}

\subsection{Using Tactile Features During Non-Visual Navigation}

Participants suggested that visually impaired people extracted tactile cues out of almost anything. Many of the textures people use for non-navigation were not specifically installed for that purpose. This appropriation of available resources has been previously recognized in nonvisual navigation accessibility literature [16, 17, 27, 41, 46].

Encountering natural guiding elements has also been studied by urban theorists such as Lynch [28] in the seminal work of image of the city. They have classified elements into (1) discrete with examples like a tree, bench, signposts etc., and (2) continuous with a fence, channel drain, hedges, etc. It can also be understood that discrete landmarks are only available from time to time and they can be either tangible or intangible (like the smell of a bakery, air current in a block, etc). Furthermore, these natural guidings can be understood as either 1) fixed elements 2) semi-fixed elements or 3) non-fixed elements as the built environment continuously changes [31].

As these natural environmental elements are important for nonvisual navigators, it would be necessary to tackle the environment to be more communicative. Particularly towards making intangible landmarks more tangible and ensuring consistency due to non-fixed perceptual cues from the environment. Finally, where effective natural environmental cues are not available, designed solutions maybe implemented to avoid gaps along a guided route.

Across the board, our survey respondents (P\#s), and all 7 blind interviewees hailed the importance of tactile feedback to nonvisual navigation. $\mathrm{O} 1$ pointed out that detecting tactile feedback is a primary purpose for using a white cane which, while in use, maintains consistent contact with the ground and nearby physical features. This provides a continuous type of tactile perception enabling key components of navigation including alerts, alignment, orientation, and confirmation.

"When you have enough experience with the cane, and enough training, you can tell the difference between a poured concrete sidewalk versus an asphalt tarred street. So you can tell those texture changes. Usually, most tactile cues can be used to a traveler's advantage as far as if I'm walking in a shopping mall and I enter into a store, the hallway of that mall may have been tiled surface. When I walk into that store, I might notice a change to a wood floor or a carpet floor. When I'm walking down the sidewalk and I approach a corner, there's going to be some sort of a texture change. Those texture changes aren't universal but there is a texture change to be found and it's good to know you're at the corner, you don't want to walk into the street." - $\mathrm{O} 1$
3.2.1 Alignment, Orientation, and Confirmation. These textures also helped our participants align themselves in different areas like the library with sidewalks, parking lots, and random seating areas. The presence of both natural and purposefully built textured surfaces coalesce to provide feedback as to the orientation of the traveler and confirmation that they are in the right place or on the right path.

For instance, P65 states that they use "the cracks in the sidewalks to confirm I was on a sidewalk and not walking in a parking lot. I also used the grass line to confirm I was traveling in a straight path to my destination." Furthermore, P28 described using the grass and sidewalk edges to help maintain their position on a path.

P43 described using natural features to both confirm their path using known landmarks as well as to identify important temporary changes. "... a planter, or bench or metal trash can become something that is looked for to know that you are traveling correctly. Also, finding a cone with a cane makes you attentive that you might find construction."

The tactile features of street crossings provide useful cues for aligning and orienting a person to the correct direction of travel. O3 teaches deaf-blind students to line up in preparation to cross a street by triangulating textures both implemented for the purposes of assisting blind people and not. For example, he pointed out that their hand placed on a properly-installed accessible pedestrian signal pole can give a general direction of travel. At the same time, they can align their feet up so they are parallel with the line separating the sidewalk from a wheelchair ramp's descension and perpendicular to the curb. This alignment, O3 explained, starts the deaf-blind person walking straight across.

T5 mentioned that many street corners she encounters are not 90-degree angles, making textured surfaces particularly useful. b

"[city] streets are weird. They sometimes come together at strange angles, like a 5-year-old drew a city.... I think the arrows [on APS]...can be helpful." -T5

T3 noted that tactile pathways, apart from providing alerts and alignment, can help reduce circumnavigation, increasing efficiency toward reaching high-traffic destinations, which require traversing large open spaces. These examples taken together indicate that the alignment and orientation of tactual features play a crucial role in the navigational abilities of our participants.

\subsection{Cautious Use of Surface Textures for Navigation}

Overall, survey respondents and interviewees while indicating many potential benefits of tactile pavings also discussed the need 
for a level of caution when using. This was especially apparent for instructors and public access experts.

3.3.1 Don't Trust the Texture Alone. Interview participants (O's \& T's) were cautious about trusting textured surfaces and suggested triangulating them with other cues. This caution was so pronounced that all five O\&M instructors explicitly taught their students to not trust them. To these instructors, textured surfaces could provide alerts, quick confirmations of correct travel, or caution of incorrect travel choices. O5 collectively referred to these elements as "something different". However, the textures themselves do not provide detailed or holistic information. In other words, O5's, and all of the O\&M instructors' students were encouraged to use other clues to determine what the "something different" actually was.

O5 explained a common scenario she encountered during lessons, "I've noticed them at parking lots and I've noticed students pick up those [textured surfaces] and think they're at a street and they count that as a street. It's not consistent enough." T5 corroborated the mixed messages textured surfaces could send.

"We have a bus station in [city] that has all these truncated domes everywhere and I think it's supposed to show it's the edge of the curb don't go off the curb but if you'd don't know, is it to show this is a place to cross? There can be some ambiguity." -T5

This ambiguity also emerged from a portion of survey participants $(n=11)$ who agreed that tactile cues were either misplaced or inconsistent. For example,

"Recently, I shorelined along an unfamiliar street. Much to my surprise, there was a random tactile mat in the middle of the block. It served no particular purpose and didn't denote anything specific. Yes, there was a corresponding tactile mat on the opposite side of the street; in the middle of the block." - P27

To combat these inconsistencies, O1, for example, taught students to get a feel for how long blocks are, or the distance of a useful chunk breaking up their route, and be mindful of how long it takes. "If the distance versus time ratio isn't making sense to them, they might be at a parking lot entrance rather than the next street corner or other important landmark."

3.3.2 Inconsistent or Incorrect Installation Leads to Errors. Inconsistent and incorrect installation of textured surfaces, at worst, could lead people to cross intersections into moving traffic. Several interviewees $(\mathrm{N}=5)$ pointed out that whether or not textured surfaces are meant to provide alignment assistance, many blind people interpret a bumpy textured surface as an indication of a safe place to cross. However, many textured surfaces are placed inside wheelchair ramps that do not line up with the crosswalk or worst, point a diagonal trajectory across the intersection. Further, two interviewees (O5 and $\mathrm{T} 5$ ) had encountered specific instances where they noticed the tactile arrows on APS poles were pointing in the wrong direction. $\mathrm{T} 3$ and $\mathrm{O} 3$ explained these inconsistencies were so pervasive and general street crossing support so poor that many deaf-blind people only travel routes vetted by an O\&M instructor. "Every route that a person learns has to be vetted for successful or ineffective installation. Maybe when there's an ineffective installation that person is not able to use that crossing." But inconsistent and improper installation could impact more than blind and deaf-blind people. T4, who reviews engineering plans for crosswalk redesigns and evaluates related $\mathrm{ADA}$ violations explained that such hazards may also endanger everyone.

"There are a few larger buildings that installed these [textured surfaces] improperly. They actually had this little lip that could come up half an inch. People were tripping over them. They were coming up off of the sidewalks." -T4

We note this difference in caution between our survey and interview respondents. While an interview offers more time for longer answers, we also learn from their expertise; people may make incorrect assumptions about the utility of textured surfaces, or they may be installed incorrectly. Such errors could lead to danger, and thus keeping safety at the forefront of our work is even more important.

\section{PRIORITIES FOR MULTIMODAL FEEDBACK THROUGH GUIDING SURFACES}

We asked participants to discuss ideal new textured surfaces in terms of what properties they would have, what information they would convey, and in what situations or contexts they would be most helpful. We discuss these results as they relate to materials, feedback types, and contexts of implementing new textures.

Participants outlined several opportunities to provide multimodal feedback in addition to improving the tactile feedback options. The majority of these solutions combine the use of additional smart devices with audio and haptic feedback to obtain more information about the environment. We present findings regarding each, as well as suggestions for additional tactile feedback.

\subsection{Desire for Mixed Materials}

Descriptions of specific properties of textured surfaces' materials were mentioned in several survey responses $(\mathrm{N}=8)$. The material focus was mainly a juxtaposition between hard vs. soft surfaces, with participants either expressing preferences towards one type of surface or utilizing both types of surfaces to aid in surface differentiation. For example,

"Perhaps something slightly squishy, like foam, versus hard textures, that could signal where doorways into buildings are, or important paths off shooting from a main thoroughfare." -P65

Over half of the responses that specifically mentioned materials envisioned surfaces with soft textures (e.g. rubber, foam). One participant, P66, specifically described their rationale behind their choice being that potentially softer surfaces that denote the presence of "any type of water surface (e.g. pool, lake, river, ocean, fountain) would help to prevent falls, injuries, and potential drownings". P27 emphasized the need for environmentally friendly materials, specifying how "upcycling would be ideal and low maintenance helps keep things cost-effective."

\subsection{Audio Feedback}

We categorized audio feedback into two types: sound feedback and voice feedback. Ten responses mainly focused on sound as a feedback mechanism either embedded into the textured surface or 
the navigation tool. For instance, P19 envisioned "a surface which made a particular sound when tapped with a white cane or made a distinct sound from the normal impact of feet on sidewalks or surrounding surfaces", while P3 described incorporating audio into the white cane to indicate other travelers or hazards.

Twelve responses focused primarily on potential voice prompts either embedded as a response to encountering the textured surfaces directly, entering environments with textured surfaces (e.g. an area surrounding the surface, such as streets and intersections), or to support proper and safe directionality.

The two categories of audio feedback ultimately converged on an overlapping topic of safety; that is, nine participants described scenarios where audio feedback can help them, for example, become more aware of other pedestrians, when to cross the street, and how to reach the other side.

\subsection{Haptic Feedback}

Similar to audio, the use of haptics was described in terms of use with a smartphone and the textured surface itself. The broader application of haptics to the Deaf and hard of hearing community was also mentioned. Participants $(\mathrm{N}=8)$ discussed haptic feedback (mainly vibration) as a feedback mechanism in response to the use of a tool such as a white cane to support crossing the street (P64). P6 described the use of haptics for encounters with textured surfaces, and the identification of specific landmarks.

"Ideally, haptic feedback could be integrated, so perhaps the bumps could vibrate when it detected an approaching phone utilizing a travel app that was meant to likewise detect the warning bumps, or only the phone could vibrate in response." -P6

Four responses expressed a preference for haptic feedback due to concerns for the hard of hearing and Deaf community.

"I think by adding a vibrating alert it would be another layer of protection... I have met many other people like myself who are not only blind but also hearing impaired. Therefore, I feel the vibrating stimulation would be more impactful than one that is auditory." $-\mathrm{P} 66$

\subsection{Visual Appearance and Aesthetics}

Another aspect for consideration was aesthetics and how it affected social attitudes. T3, who has experience consulting corporate as well as government-funded projects, noted the importance of satisfying access needs while maintaining the desired look and feel, an intersection she believed actually fostered more creativity. Throughout her advocacy, T2 has seen this in practice; she has used a municipality's choice to renovate their downtown sidewalks to maintain dark red brick while ensuring high-contrast bright white brick was used to denote street corners. In this case, providing a warning of street corners did not come at the expense of preserving history.

Many tactile guiding surfaces are already "multimodal" as they are often designed with high-contrast colors to support low-vision navigation. T3 explained the many factors that must be considered when designing the visual appearance of textured surfaces both to make them more aesthetically pleasing as well as low vision accessible, especially in outdoor conditions.

Beyond the specific types of feedback, the visual presentation of textured surfaces could have an effect on how passersby code the purpose of the textured surfaces and blind people. T3 noted that it was important for her blind stakeholders that they are not perceived as walking on the 'blind path.' Though none of our interviewees experienced this directly, they shared stories of their students and blind friends experiencing public humiliation by passersby if they were not walking on textured guide paths. Whereas passersby may have thought they were being helpful to point out the existence of a guide path, $\mathrm{O} 2$ and $\mathrm{T} 5$ mentioned this assistance became patronizing if a blind person did not prefer to use it or was going somewhere different from where the path led.

Relatedly, O4 experienced frequent patronizing and sometimes misgendering assistance while reading braille signs. Passersby would suggest that whichever bathroom sign they were reading, for example, was either the right or wrong place where they should use the restroom, raising important awareness about the deeply personal nature of, and the longstanding discriminatory histories such assistance may involuntarily bring a user's attention to. T3 believed that if textured surfaces are ultimately meant to be interacted with up close, thus probably engendering unwanted attention, textured surfaces that took on the aesthetic of the environment and provide utility for more than blind people may help to destigmatize sticking to, or not sticking to, a particular path.

While we acknowledge the insufficiency of secondhand accounts, this reminder hints at specific considerations when designing and evaluating textured surfaces to, as researchers have advocated for years, consider their effect on passersby and their social accessibility.

\subsection{Optimize Feedback to Reduce Cognitive Demand}

With the promise of multimodal feedback, there also comes the concern of too much information. Interviewees were enthusiastic about textured surfaces outfitted with haptic and audio feedback but noted that it must be implemented thoughtfully. Five participants expressed concerns about distractions that may arise from navigators needing to pay attention to too many signals. O5 listed several potential sources of information a student may be triangulating, and cautioned these sources could be many, underscoring the need for information sources to be voluntarily activatable if not sharing the more pertinent information.

"The con is you have something talking to you plus you're listening to traffic plus you're trying to feel truncated domes plus you're trying to feel an arrow. Some students, in particular, can get really distracted by all of that information." - O5

There was concern that stepping on a vibrating surface, for example, would alarm some people; and even if activatable, if others could be standing on it and unaware, they could still be alarmed. Whereas expecting people in public to traverse textured surfaces seemed reasonable, interviewees cautioned that other types of feedback should be voluntarily activated, and haptic feedback may be best transmitted through poles $(\mathrm{O} 3)$. 


\subsection{Placement of Future Textures in Public Spaces}

All participants were generally positive about the idea of new textured surfaces to support nonvisual navigation. While survey participants generally provided speculative future uses of novel surface textures, interviewees first wanted existing textured surfaces that are known to be helpful to be installed in more places. For example, several noted $(\mathrm{N}=37)$ that textured surfaces might be installed near crosswalks of busier streets and in newly renovated or constructed areas, but much of the built environment lacks them. They suggested that amplifying existing textured surfaces that are known to be useful is an important first step to supporting tactile feedback during nonvisual navigation.

4.6.1 Public transit stations. Seven survey participants specified the need for more effective organization of tactile cues related to transportation, either regarding approaching transportation stops or traveling within large transportation hubs (e.g., train stations, airports). All seven blind interviewees reported that bus stops are almost always difficult to find given their inconsistent features and how difficult transit stations are to traverse. T1, who gained extensive knowledge about his city's public transit by working at the agency and teaching people to use it described a time when he waited in the wrong place since the bus stop was not tactually different. "I sat there and I heard the bus come by. Well, it passed by. Come to find out, I was standing at a speed limit sign. [T1]" T1 and others shared that even experienced travelers still encounter difficulties finding where to board public transit and noted it a fruitful place to innovate on textured services since there may not be sufficient environmental clues to take advantage of. P54 suggested

"I'm picturing a textured surface with ridges, made of metal or plastic, that could be easily detected with a cane but would be flush with the surface of the sidewalk. I could see these being very helpful at the transit center, where I need to find the exact place where each bus would stop. As I'm walking along, these exact bus stops are not easy to find, since I can't see them. These would be very useful for marking bus stops anywhere in the community. If a change is made in where the bus stops, they could easily be removed, and asphalt squares could be installed in their place." -P54

This story is reminiscent of many others [12, 41], but many interviewees really wanted to tactually discern bus stops and preferred to learn only more complex information through smartphone apps, the solution proposed in this prior work. O3, for example, believed poles at textured surfaces marking where train doors would open would be excellent places to provide localized vibration and audio feedback for on-demand updates about next arrivals.

4.6.2 Mixed-use public places. Mixed-use public places are areas where people traverse near different types of mobility aids (pedestrian, biker, scooter, vehicle). Three interview participants explicitly noted new construction in their areas that contained no tactile boundaries between these spaces in the original design. While meant to ease movement and emphasize non-vehicle modes of transport by, for example, narrowing streets to make room for bike lanes, the proliferation of these tactually ambiguous mixeduse zones was concerning to interviewees. From O3's advocacy, he noted textured surfaces installed as an afterthought to provide some boundaries need to be particularly prominent as the consequences of missing them could put pedestrians in danger. However, if the texture too much resembles that at crosswalks which participants found quite detectable (like T5's prior-quoted concern), they could pose an additional danger by being interpreted as a safe place to cross. On one project, $\mathrm{O} 3$ noticed large planters replacing a textured surface determined not detectable enough by blind stakeholders. The blending of traffic in mixed-use movement zones provide an opportunity to re-emphasize early involvement so textured surfaces and appropriate barriers are robust and well incorporated into the landscape.

\section{PRACTICAL CONSIDERATIONS FOR IMPLEMENTATION}

Interviewees and survey respondents shared important factors to consider during the design of textured surfaces which align with several features of good design. As textured surfaces tend to be found in the built environment, however, these suggestions not only concerned the surfaces themselves but the entire process of their design, installation, movability, and learnability. The latter three phases have been addressed more sparingly in accessibility research. To elucidate these suggestions, participants often shared their experiences encountering or collaborating on projects with various successes and failures.

As mentioned, inconsistency in the environment created frustration and mistrust in participants; any textured surface must have a consistent design and implementation. This suggestion was so prominent as highlighted by T5, adhering to it could transform the guesswork of interpretation, "Tactile feedback could play a much bigger role if it were to be used consistently. (T5)" For example, even if textured surfaces are designed according to best practices, they may still be installed incorrectly. This raises the need for fabricators to take on advocacy and scaffolding work to help ensure partners in augmenting the built environment do not morph what could provide useful feedback into yet another distraction.

\subsection{Standardization}

Throughout our study participants described issues with inconsistent and incorrect installation and use of purposefully installed textures for navigation. We reiterate the importance of standardization to address these issues while maintaining that the process for doing so may not be as straightforward as practical constraints and multiple stakeholders can make coordination difficult. Participants highlighted the significance of standardization regarding materials: "There needs to be national standards, that is, using the same system everywhere so they are uniform" (P52). Such standards do exist however their enforcement may be far less universal as P63 explains:

"I don't believe that we blind and [visually impaired] need yet another tactile surface for navigation. Just like with legislators, we don't need more laws - we need laws standardized, funded, and enforced. I urge 
you to develop and implement a standardized set of tactile interfaces that are globally agreed to and applied. Funding, then, is directed toward production, implementation, servicing, training the trainers, and training individuals." - P63

\subsection{Installation}

Interviewees noted that installation must be done properly and consistently for users to benefit the most. At best, inconsistencies produced unwieldy searches like T1's experience at hotels, "If you go into any building the braille sign is never in the same place as another building. For instance, I can go to a hotel and the room number may be on the door or the left side of the door, or the right side of the door. So you never know. You have to feel around for it." At worst, however, as mentioned previously, incorrect installations could lead users into the middle of intersections or even become tripping hazards.

T3 spoke of another project that sounded good in theory, but insufficient feedback loops meant a test implementation went unverified, rendering the textures useless.

"We said we need signs, and these signs need certain criteria and they need braille, raised print, and raised arrows but how do the people find the signs? We created this ground texture, and then we agreed on that ground texture with blindness stakeholder groups. But when they put the stuff down you couldn't feel them well so they did it, and then they didn't really have us back to check it in a timely way. So it was awful because I went and looked and felt, and this whole team was all feeling and we couldn't really feel the difference between the walkway and the indicator to tell folks where the sign was." -T3

As part of her consulting, T3 aims to get her clients to engage stakeholders in tight feedback loops, long advocated by accessibility research. Apart from their reminder to more deeply engage stakeholders, these shortcomings point to opportunities where accessibility researchers might incorporate these cautions and collaboration best practices during installation, not just prototyping.

\subsection{Movability}

O3 was our only interviewee who had extensive experience designing accessible pathways through an ever-changing landscape: a machine shop that employed several blind people. However, we believe his wisdom is useful for temporary contexts. For decades, the machine shop comprised pathways made of thermoplastic that had to be ground down whenever the shop design changed, and the pathways needed to be redrawn. This process became unwieldy as the shop floorplan changed frequently. They have since transitioned to using metal plating like that found covering underground access points along sidewalks. O3 mentioned the metal material would be inappropriate to use outdoors as they are a slipping hazard when wet, but for their indoor shop, it can be screwed into and unscrewed from the concrete flooring with available tools, is easy to procure, and provides sufficient tactile and audio feedback for deaf-blind and blind employees to travel efficiently. Whereas metal may be inappropriate in some indoor environments, the lesson to think of the possibilities textured surfaces may need to be moved if they are used for pop-up events like poster sessions at conferences, or if bus stops are redistributed, while choosing durable materials that do not move accidentally is important.

\subsection{Discoverability and Learnability}

Almost all interview participants appreciated the potential for textured surfaces but acknowledged that learnability is an ongoing challenge. This sentiment was echoed by survey participants who broadly expressed a desire to be more well versed in the meaning of existing textures and tactile features. Some, like O3, recommended locator tones like those used to denote the nearby presence of an APS; and O4, among others, recommended that tactile feedback could be outfitted with sensors that alerted smartphone users of their whereabouts to limit the environmental noise. T3 and T5 described how websites and audio announcements could be used to provide travelers with instructions for how to locate accessibility features like tactile maps and signs. Additionally, connections, or lack thereof, could impact discoverability, learnability, and ultimately, utility. $\mathrm{O} 3$ pointed out the consequences of helpful textured surfaces that fail to connect their users to a point where they can pick up other helpful cues when the textured surface ends. To exemplify this, he recounted a project gone wrong:

"It's important that the [textured surface] intersects with the likely path.... The [textured surface] wasn't extended from the station out to the streetscape, so there was no wayfinding material to assist someone from the station through the plaza which has a bunch of street trees on the side of it in raised beds that working that plaza is difficult or impossible for someone who doesn't have experience already. ... They need to think about the connection between the [train] boarding and the streetscapes. People need to be able to get out of the station to get on their way." -O3

O3's anecdote demonstrates the importance of thinking about the before and after when designing textured surfaces; in what ways may the user be encountering them, and in what ways should the textured surfaces support them on their way? The misstep of not connecting a textured guide path along a train station's platform to a nearby street with well-defined sidewalks rendered the textured surfaces less discoverable, and therefore, less useful than they could have been.

The most consistent request from interviewees was for more tactile feedback to denote the presence of bus stops and to provide guidance in public transit stations. They noted discoverability in these scoped locations may be easier because of consistent station layouts in some cases or consistent features, like stairways and elevators, such that a common language could be established. If someone can find one landmark detectable by a cane, they may then know how to orient to additional feedback.

\subsection{Need for Transparency in the Process}

Finally, discussions with public transit experts revealed the importance of transparency in the process of producing and implementing textured surfaces for navigation. The four disabled accessibility 
experts (T1, T2, T3, T5) noted that processes for implementing textured surfaces and other accessibility features in public remained opaque. There continually is confusion about how citizen-reported ADA violations were determined to be actual violations or to be feasibly fixable or not (T2) and the reasons behind decisions that insufficiently outfit new projects with accessibility features (T1, T2, T3, T5). To these public accessibility experts, communication among stakeholder groups was insufficient. T5 echoed that these problems even existed inside of the transit agency where she worked.

"When other departments in the system decide to make changes, they are supposed to consult us. A lot of times they did but there were always those times that stuff happened that we didn't know about which was very annoying and frustrating." -T5

T4 outlined the various stakeholders involved in implementing public accessibility features: an ADA coordinator, the engineer who reviews plans, funders, a project contractor, construction workers, and pedestrians. The ADA coordinator liaises with external stakeholders who may or may not be consulted for feedback proactively depending on whether the agency thinks the potential solutions are unclear.

T4 further admitted that though his municipality's interpretations of the ADA formed standards are publicly viewable online, many complicated design decisions and negotiations are often handled on phone calls. This leaves few traces about how such decisions are made for public critique, revision, or, in our case, design inspiration, manufacturing process, and tool development guidance.

\section{DESIGN RECOMMENDATIONS FOR MULTIMODAL FEEDBACK ENABLED NAVIGATION TILES}

We have thus far reported factors and situational contexts that textured surface designers and practitioners must consider from multiple perspectives that include blind people, O\&M instructors, and public accessibility experts. In this section, we overview the design recommendations for key technologies from stakeholders' perspective to alleviate the challenges that non-visual navigators face when interacting with textured surfaces. We outline our proposed recommendations to enable the creation of low-cost, customizable, multi-modal textured surfaces. Our design recommendations are broadly situated over three areas: democratized production of tactile surfaces, multimodal sensing, and feedback, and support for planning and installation.

\subsection{Democratized Textured Surface Production}

6.1.1 Open process for consistency. Due to the cost and complexity of the current production methods, there is a high barrier for further tactual exploration and physical validation of consistent standards in textured surfaces. To enable a wide variety of textures and to validate the consistency, a prototyping method should be open and accessible to teachers and other stakeholders. Current methods, unfortunately, do not afford such exploration, precision-engineered mold takes 2-3 weeks to be machined before it is used to mold a textured surface. By incorporating rapid prototyping technologies such as $3 \mathrm{D}$ printing for making molds, the time to prototype and explore newer textures is drastically reduced. Having a low-cost customizable production model also enables stakeholders to quickly understand what is feasible, which may enable tighter feedback loops to address issues with surface installation.

6.1.2 Support new materials \& textures. Further, by having an opensource process, new material explorations are possible for many of the textures desired by our survey participants as illustrated in Section 4.2 and 5.1. For example, textured surfaces provided participants important information, but their presence and meaning weren't always discoverable. Different texture shapes were often too similar to draw participants' attention to their differing meanings. To be discoverable, textures should have a contrast with the surrounding environment such as hard guidance surfaces on otherwise soft surfaces \& materials. Furthermore, research in advanced materials [19] has identified texture changing polymers that could be incorporated into surface production. While a lot of mixed material prototyping is possible and HCI research may recommend new designs, we should collaborate more with materials, civil, and other engineers to confirm that the safest, most durable, and feasible materials are being used.

\subsection{Multimodal Feedback Sensing and Feedback}

While current textured surfaces are often manufactured as singular modes of information (tactile), we learned, as well as others [41, 46] that many feedback sources are combined to inform navigation decisions. Further, textured surfaces were often misunderstood which could lead to incorrect or even dangerous navigation. In many of our interview and survey responses, participants alluded to either not recognizing the textures or detecting them and being led to the wrong location.

Participants perceived the benefits of multimodal feedback to complement textured surfaces that could assist in learnability and appropriate use. This is consistent with our survey participants supporting the idea of audio or haptic feedback with the preference to receive the audio through their personal devices and further control of activating such messaging. With low-cost conformal electronics material such as pressure-sensitive textiles, a system could detect user input before delivering audio feedback. Audio feedback could be provided as prompts or voice feedback, as desired. Further, audio feedback could be continuous (e.g. as turn-by-turn directions) or intermittent.

6.2.1 Support Multimodal Feedback Customizability. However, we note that multimodal feedback should be customizable to prevent information overload. As participants also describe the need to minimize or control information flow to avoid information overload while navigating. NavTiles should allow users to customize when they receive feedback, control of the frequency, and duration of voice prompts should lie with the user. NavTiles should support users to receive information on their own devices than public infrastructure (such as poles, sidewalks, etc.) to avoid confusing nonusers. 
6.2.2 Supporting learnability \& discoverability. While navtiles should be implemented at obvious stopping points (e.g. crossings, intersecting paths), multimodal feedback (audio+tactile) could be used to support the discoverability and learnability of new and existing textures by providing detailed information such as transit stop names, different paths, or pointers to other landmarks.

\subsection{Consistent Installation, Deployment and Resource Planning}

6.3.1 Support consistent standard implementation. Having an overview of where to implement tiles (in the built environment) helps resource planners and implementers might lead to a more consistent implementation outcome (see Section 4.3.2). Hence software for multimodal tiles should support an overview interface to track and plan tile locations. Finally, existing standards do not address multimodal feedback and are not followed consistently, as evidenced by participants encountering surfaces with inconsistent meanings. In the planning app, these standards could be designed, incorporated as templates and warnings can be implemented when a user deviates from the design. Finally, the toolkits \& software used to prototype NavTiles could incorporate information about required approvals before installation. Future research should work in collaboration with materials, civil engineering, and policy experts to refine and distribute standards.

6.3.2 Support movability. Besides installation, another key area is to help support movability or constant environmental changes around where tiles are deployed. This means that when tiles are moved, their meanings may change; one way to accommodate that is by enabling implementers to keep track of which tile is deployed where and enable them to update audio instructions for the tile feedback or prompts. The potential portability and multimodal feedback of NavTiles may also be applicable in temporary setups, such as street fairs, where permanent infrastructure may be infeasible. For example, interviewees mentioned textured surface movability was imperative when workstations in a shop changed.

6.3.3 Support Diverse \& Safer Involvement in Future Research. While the textured surfaces are typically implemented by governmental agencies, if there are delays or lapses in this installation, this open, low-cost process may enable a broader \& diverse audience to deploy custom textured surfaces to fill gaps in the system. Partnership with blind people, especially deaf-blind people who may rely even more on tactile feedback, may help reduce the inconsistent textured surfaces that confused participants. Future research should also be cognizant of successful navigation techniques to complement, without overshadowing, other sensory information. Finally, we should use existing safe spaces as testbeds for innovation. Due to safety concerns related to testing material durability and confusion over textured surface meaning outlined by participants, prototype technologies must be deployed responsibly with clear boundaries first.

\section{A PROTOTYPE IMPLEMENTATION}

The research team conducted exploratory prototyping activities to develop a process for producing textured surfaces with embedded sensors, capable of providing multi-modal feedback to non-visual navigators. This consisted of three components: planning, fabrication of tiles, and embedding of interactive elements. All of the components presented for the NavTiles prototype system (web app, hardware, etc.) have been completed and tested for functionality. Future work will involve testing of the prototypes with blind people and initial deployments.

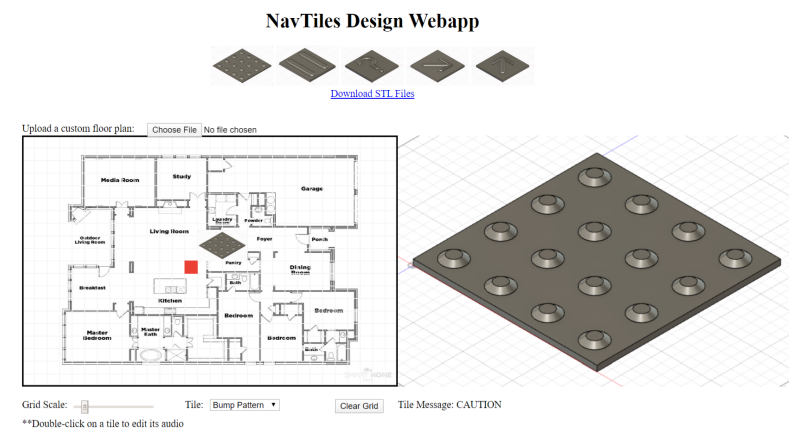

Figure 3: Shows a web application UI for custom planning of the tiles and recording audio prompts. This application is running on a web server and is sending audio prompts to users phone

Planning Application: Users can plan where they want to instrument a navigation tile in the environment using a planning app, where custom layouts can be uploaded and tiles with different textures can be placed with corresponding audio prompts. On deciding the type of tiles, users can download the necessary files to produce $3 \mathrm{D}$ textures. The planning app is shown illustrating an indoor installation in Fig 3.

Fabricating a NavTile: To make a textured surface, users can $3 \mathrm{D}$ print the texture elements using a wide variety of materials. Desktop 3D printers with low-cost thermoplastic materials are available as low as $\$ 99$. While a one-off custom designed NavTiles could be 3D printed tile (as seen in Fig 4A) can be used as-is, the original could be used as molds for more replication. For instance, the printed objects can be used to make several copies using a low-cost DIY vacuum former (Figure 4A).

Integrating Sensing Assembled tiles can then be interfaced with textile pressure sensors (costing $<\$ 2$ per m2) called VeloStat to detect a user's step. These pressure sensors are connected to an ESP32 IoT device which is run on a battery (see Fig 4C). The pressure sensors are simply attached to the back of the tiles, as a user steps on the tile, the change in resistance is detected by ESP32 to trigger the audio.

Networked Connection and audio In order to uniquely identify the user, we can receive a Bluetooth signal from the user's phone. As soon as the user interacts with the tile, a request is sent to the webserver from internet-connected ESP32 through the MQTT protocol and audio of pre-recorded message for the corresponding tile is played to the user's phone.

\section{CONCLUSION}

In this paper, we investigate the role of tactile cues through a mixedmethods study with blind travelers, O\&M instructors, and public 


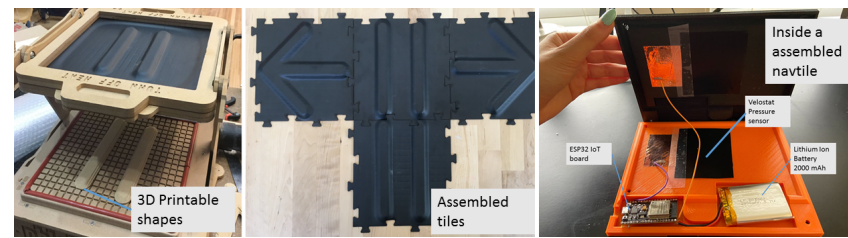

Figure 4: (A) Shows 3D printable textures and a thermoformer for making a texture, (B) Shows guidance surfaces inter-locked with each other (C) Underlying electronics, a sensor to make the tiles multi-modal.

accessibility experts. Our study uncovered the role of tactility in everyday navigation and attitudes surrounding the use of tactile guidance surfaces for non-visual navigation. Our findings indicate that tactile cues although are very important and useful remain inconsistently applied in the built environment and potential breakdowns happen.

Further, our findings reveal several opportunities envisioned by participants for augmenting the existing tactile surfaces with multi-modality, i.e., not just tactile alone. The perception from each stakeholder group is of cautious optimism while being open to new advances in multi-modal textured surface designs. We further discuss implementation challenges expressed by our participants such as standardization, installation, movability, discoverability, and a need for transparency in any new design alternative.

Finally, we offer a potential approach for rapidly creating lowcost multi-modal textured surfaces with easily available materials and tools to widen the processes of production. We argue that new design alternatives alone are not enough to support nonvisual navigation but pragmatic implementation complemented with insights from key stakeholder, the context of use is needed for long term change.

\section{ACKNOWLEDGMENTS}

We thank the participants of the study. We would like to acknowledge undergraduate students Shiva Peri and Nali Huynh for their help in developing prototypes. We would also acknowledge Elena Deng, Simran Jobanputra, Emily Wu for their early brainstorming efforts. This research was funded in part by NSF IIS-1718651.

\section{REFERENCES}

[1] 2020. Phlatformer v2. https://openbuilds.com/builds/phlatformer-v2.2545 [Online; accessed 16. Sep. 2020].

[2] 2020. Swell Form Machine. http://www.americanthermoform.com/product/ swell-form-graphics-ii-machine [Online; accessed 16. Sep. 2020]

[3] 2020. ViewPlus | Delivering Sense Ability. https://viewplus.com [Online; accessed 16. Sep. 2020].

[4] Dragan Ahmetovic, Cole Gleason, Chengxiong Ruan, Kris Kitani, Hironobu Takagi, and Chieko Asakawa. 2016. NavCog: a navigational cognitive assistant for the blind. In Proceedings of the 18th International Conference on Human-Computer Interaction with Mobile Devices and Services. 90-99.

[5] Tomohiro Amemiya, Jun Yamashita, Koichi Hirota, and Michitaka Hirose. 2004 Virtual leading blocks for the deaf-blind: A real-time way-finder by verbalnonverbal hybrid interface and high-density RFID tag space. In IEEE Virtual Reality 2004. IEEE, 165-287.

[6] Paul Arthur and Romedi Passini. 1992. Wayfinding: people, signs, and architecture

[7] P Barker. [n.d.]. Barrick,). and Wilson, R.(1995) Building Sight: A Handbook of Building and Interior Design Solutions to Include the Needs of Visually Impaired People.

[8] Billie L Bentzen, Tina Nolin, Randolph Easton, et al. 1994. Detectable warning surfaces: color, contrast, and reflectance. Technical Report. United States. Federal Transit Administration.
[9] Michael Brambring. 1985. Mobility and orientation processes of the blind. In Electronic spatial sensing for the blind. Springer, 493-508.

[10] Virginia Braun and Victoria Clarke. 2006. Using thematic analysis in psychology. Qualitative research in psychology 3, 2 (2006), 77-101.

[11] Erin Buehler, Stacy Branham, Abdullah Ali, Jeremy J Chang, Megan Kelly Hofmann, Amy Hurst, and Shaun K Kane. 2015. Sharing is caring: Assistive technology designs on thingiverse. In Proceedings of the 33rd Annual ACM Conference on Human Factors in Computing Systems. 525-534.

[12] Megan Campbell, Cynthia Bennett, Caitlin Bonnar, and Alan Borning. 2014. Where's my bus stop? Supporting independence of blind transit riders with StopInfo. In Proceedings of the 16th international ACM SIGACCESS conference on Computers \& accessibility. 11-18.

[13] Patrick Carrington, Shannon Hosmer, Tom Yeh, Amy Hurst, and Shaun K Kane. 2015. " Like This, But Better": Supporting Novices' Design and Fabrication of 3D Models Using Existing Objects. iConference 2015 Proceedings (2015).

[14] PK Edman. 1992. Tactile Graphics, American Foundation for the Blind.

[15] Stéphanie Giraud and Christophe Jouffrais. 2016. Empowering low-vision rehabilitation professionals with "do-it-yourself" methods. In International Conference on Computers Helping People with Special Needs. Springer, 61-68.

[16] Nicholas A Giudice. 2018. Navigating without vision: Principles of blind spatial cognition. In Handbook of behavioral and cognitive geography. Edward Elgar Publishing.

[17] Reginald G Golledge, Roberta L Klatzky, and Jack M Loomis. 1996. Cognitive mapping and wayfinding by adults without vision. In The construction of cognitive maps. Springer, 215-246.

[18] João Guerreiro, Daisuke Sato, Saki Asakawa, Huixu Dong, Kris M Kitani, and Chieko Asakawa. 2019. CaBot: Designing and Evaluating an Autonomous Navigation Robot for Blind People. In The 21st International ACM SIGACCESS Conference on Computers and Accessibility. 68-82.

[19] Mark Guttag and Mary C Boyce. 2015. Locally and dynamically controllable surface topography through the use of particle-enhanced soft composites. Advanced Functional Materials 25, 24 (2015), 3641-3647.

[20] Kotaro Hara, Vicki Le, and Jon Froehlich. 2013. Combining crowdsourcing and google street view to identify street-level accessibility problems. In Proceedings of the SIGCHI conference on human factors in computing systems. 631-640.

[21] Leona Holloway, Kim Marriott, and Matthew Butler. 2018. Accessible maps for the blind: Comparing 3D printed models with tactile graphics. In Proceedings of the 2018 CHI Conference on Human Factors in Computing Systems. 1-13.

[22] William Henry Jacobson. 1993. The art and science of teaching orientation and mobility to persons with visual impairments. American Foundation for the Blind.

[23] Dae Shik Kim, Robert Wall Emerson, and Eleni Gaves. 2016. Travel in adverse winter weather conditions by blind pedestrians: effect of cane tip design on travel on snow. Fournal of visual impairment \& blindness 110, 1 (2016), 53-58.

[24] Andreas Kunz, Klaus Miesenberger, Limin Zeng, and Gerhard Weber. 2018. Virtual navigation environment for blind and low vision people. In International Conference on Computers Helping People with Special Needs. Springer, 114-122.

25] Steven Landau and Karen Gourgey. 2001. Development of a talking tactile tablet. Information Technology and Disabilities 7, 2 (2001)

[26] Anatole Lécuyer, Pascal Mobuchon, Christine Mégard, Jérôme Perret, Claude Andriot, and J-P Colinot. 2003. HOMERE: a multimodal system for visually impaired people to explore virtual environments. In IEEE Virtual Reality, 2003. Proceedings. IEEE, 251-258.

[27] Jack M. Loomis, Roberta L. Klatzky, and Nicholas A. Giudice. 2013. Representing 3D Space in Working Memory: Spatial Images from Vision, Hearing, Touch, and Language. DigitalCommons@UMaine (2013), 131. https://doi.org/10.1007/978-14614-5879-1_8

[28] Kevin Lynch. 1960. The Image of the City. Boston: Mass, EE. UU.

29] Tomomi Mizuno, Arisa Nishidate, Katsumi Tokuda, and ARAI Kunijiro. 2008. Installation errors and corrections in tactile ground surface indicators in Europe, America, Oceania and Asia. IATSS research 32, 2 (2008), 68-80.

[30] John Parkin and Nicola Smithies. 2012. Accounting for the needs of blind and visually impaired people in public realm design. Fournal of urban design 17, 1 (2012), 135-149.

[31] Amos Rapoport. 1990. The meaning of the built environment: A nonverbal communication approach. University of Arizona Press.

[32] Timm Rosburg. 2008. Tactile ground surface indicators in public places. In Human Haptic Perception: Basics and Applications. Springer, 491-499.

[33] David W Schloerb, Orly Lahav, Joseph G Desloge, and Mandayam A Srinivasan. 2010. BlindAid: Virtual environment system for self-reliant trip planning and orientation and mobility training. In 2010 IEEE Haptics Symposium. IEEE, 363370

[34] Alan C Scott, Janet M Barlow, David A Guth, Billie Louise Bentzen, Christopher M Cunningham, and Richard Long. 2011. Walking between the lines: Nonvisual cues for maintaining headings during street crossings. Journal of Visual Impairment \& Blindness 105, 10 (2011), 662-674.

[35] Agneta Ståhl and Susanne Iwarsson. 2007. How do blind people orient themselves along a continuous guidance route?: Summery. (2007) 
[36] Sarit Szpiro, Yuhang Zhao, and Shiri Azenkot. 2016. Finding a store, searching for a product: a study of daily challenges of low vision people. In Proceedings of the 2016 ACM International foint Conference on Pervasive and Ubiquitous Computing. 61-72.

[37] Aud Tennøy, Kjersti Visnes Øksenholt, Nils Fearnley, and Bryan Matthews. 2015 Standards for usable and safe environments for sight impaired. In Proceedings of the Institution of Civil Engineers-Municipal Engineer, Vol. 168. Thomas Telford Ltd, 24-31.

[38] Lauren Thévin, Carine Briant, and Anke M Brock. 2020. X-Road: Virtual Reality Glasses for Orientation and Mobility Training of People with Visual Impairments ACM Transactions on Accessible Computing (TACCESS) 13, 2 (2020), 1-47.

[39] Lauren Thevin and Anke M Brock. 2018. Augmented reality for people with visual impairments: Designing and creating audio-tactile content from existing objects. In International Conference on Computers Helping People with Special Needs. Springer, 193-200.

[40] Lauren Thévin, Christophe Jouffrais, Nicolas Rodier, Nicolas Palard, Martin Hachet, and Anke M Brock. 2019. Creating Accessible Interactive Audio-Tactile Drawings Using Spatial Augmented Reality. In Proceedings of the 2019 ACM International Conference on Interactive Surfaces and Spaces. 17-28.

[41] Anja Thieme, Cynthia L Bennett, Cecily Morrison, Edward Cutrell, and Alex S Taylor. 2018. " I can do everything but see!"-How People with Vision Impairments Negotiate their Abilities in Social Contexts. In Proceedings of the $2018 \mathrm{CHI}$ Conference on Human Factors in Computing Systems. 1-14.

[42] Department for Transport. 2007. Using tactile paving surfaces. GOV (Jun 2007). https://www.gov.uk/government/publications/guidance-on-the-use-oftactile-paving-surfaces
[43] Dimitrios Tzovaras, Konstantinos Moustakas, Georgios Nikolakis, and Michael G Strintzis. 2009. Interactive mixed reality white cane simulation for the training of the blind and the visually impaired. Personal and Ubiquitous Computing 13, 1 (2009), 51-58.

[44] MA Williams, B Dubin, C Amaefule, L Nguyen, A Abdolrahmani, C Galbraith, A Hurst, and SK Kane. 2016. Better supporting blind pedestrians and blind navigation technologies through accessible architecture. In Designing Around People. Springer, 237-246.

[45] Michele A Williams, Caroline Galbraith, Shaun K Kane, and Amy Hurst. 2014. " just let the cane hit it" how the blind and sighted see navigation differently. In Proceedings of the 16th international ACM SIGACCESS conference on Computers \& accessibility. 217-224.

[46] Michele A Williams, Amy Hurst, and Shaun K Kane. 2013. " Pray before you step out" describing personal and situational blind navigation behaviors. In Proceedings of the 15th International ACM SIGACCESS Conference on Computers and Accessibility. 1-8.

[47] Yuhang Zhao, Cynthia L Bennett, Hrvoje Benko, Edward Cutrell, Christian Holz, Meredith Ringel Morris, and Mike Sinclair. 2018. Enabling people with visual impairments to navigate virtual reality with a haptic and auditory cane simulation. In Proceedings of the 2018 CHI conference on human factors in computing systems. $1-14$.

[48] Yuhang Zhao, Elizabeth Kupferstein, Doron Tal, and Shiri Azenkot. 2018. " It Looks Beautiful but Scary" How Low Vision People Navigate Stairs and Other Surface Level Changes. In Proceedings of the 20th International ACM SIGACCESS Conference on Computers and Accessibility. 307-320. 